\title{
Human chondrocyte-conditioned medium promotes chondrogenesis of bone marrow stem cells
}

\author{
Rabiatul Adawiyah Razali', Yogeswaran Lokanathan², Shiplu Roy Chowdhury², Nor Hamdan \\ Mohamad Yahaya3 ${ }^{3}$ Aminuddin Bin Saim4, Ruszymah Bt Hj Idrus, 1,2,*
}

\section{Abstract}

Background: Cell-based therapy for osteoarthritis requires culturing of good quality cells, especially with a chondrogenic lineage, for implantation.

Objective: To investigate the ability of chondrocyte-conditioned medium (CCM) to induced chondrogenesis.

Methods: Bone marrow mesenchymal stem cells (BMSCs) were subjected to chondrogenic induction using CCM and chondrocyte induction medium (CIM). The optimal condition for the collection of CCM was evaluated by quantifying the concentration of secreted proteins. The chondrogenic efficiency of BMSCs induced by CCM (iCCM) was evaluated using immunocytochemical analysis, Safranin-O staining, and gene expression.

Results: Protein quantification revealed that CCM obtained from cells at passage 3 at the $72 \mathrm{~h}$ collection point had the greatest amount of protein. Supplementation of CCM results in the aggregation of BMSCs; however, no clumping was visible as in iCIM. The expression of collagen type 2 was detected as early as day 7 for all groups except for non-induced BMSCs; however, the level of expression decreased with culture time. Similarly, all tested groups showed positive staining for Safranin-O as early as day 7. The induction of BMSCs by CCM caused the down-regulation of collagen type 1, along with the up-regulation of the collagen type 2, ACP and SOX9 genes.

Conclusion: The optimum CCM to induce BMSC into chondrocytes was collected at passage 3 after $72 \mathrm{~h}$ and was used in a 50:50 ratio of CCM to fresh medium.

Keywords: cartilage; chondrogenesis; osteoarthritis; stem cells

Articular cartilage is a type of connective tissue, located at the end of the bones, which assists in joint mobility and also helps to withstand mechanical load on the joint. Defects that are caused by disease or injury are usually irreversible due to the properties of articular cartilage itself, which have a low self-repair potential [1]. One of the diseases of cartilage defects is osteoarthritis $(\mathrm{OA})$, which will cause a reduction in patient quality of life due to restricted joint movement and extreme pain. In total, $10 \%$ of men and $13 \%$ of women in the adult population aged over 60 years suffer from OA; it is a leading cause of pain and disability in most countries worldwide $[2,3]$.

\footnotetext{
*Correspondence to: Ruszymah Bt Hj Idrus, Tissue Engineering Centre, Faculty of Medicine, Universiti Kebangsaan Malaysia, Kuala Lumpur 56000, Malaysia, e-mail: ruszymah@ppukm.ukm.edu.my, ruszyidrus@gmail.com 'Department of Physiology, Faculty of Medicine, Universiti Kebangsaan Malaysia, Kuala Lumpur 56000, Malaysia ${ }_{2}^{2}$ Tissue Engineering Centre, Faculty of Medicine, Universiti Kebangsaan Malaysia, Kuala Lumpur 56000, Malaysia ${ }^{3}$ Department of Orthopaedic and Traumatology, Faculty of Medicine, Universiti Kebangsaan Malaysia, Kuala Lumpur 56000, Malaysia ${ }^{4}$ Ear, Nose and Throat Consultant Clinic, Ampang Puteri Specialist Hospital, Selangor 68000, Malaysia
}

¿ Open Access. ( 2020 Razali et al., published by Sciendo. (c) Br-Nc-ND This work is licensed under the Creative Commons Attribution NonCommercial-NoDerivatives 4.0 License. 
Cell-based treatments of OA and other cartilage defects require the culturing of human cells to reach sufficient cell numbers for implantation. However, the challenges to produce high quality cultured cells or alternative cell sources for cartilage repair and at the same time cells which are suitable for implantation still prevail. Chondrocytes produce extracellular matrix (ECM) which is rich in collagen type II, aggrecans, link proteins and smaller amounts of collagen types IX and XI [4]. However, during tissue processing and cell culture, chondrocytes may lose features and the characteristic pattern of matrix protein expression [5]. Therefore, the search for alternative methods and cell sources is still ongoing.

Other than chondrocytes, multipotent mesenchymal stem cells (MSCs) isolated from bone marrow stem cells (BMSC) and chondrogenic differentiated MSCs could be alternative sources for cartilage repair. Differentiation could be achieved by (i) the addition of growth factors and cytokines to the culture medium, (ii) co-culturing the MSCs with chondrocytes, or (iii) by means of genetic modulation [6-9].

Co-culturing of BMSCs and chondrocytes has been demonstrated to be able to enhance the chondrogenesis ability of BMSCs $[10,11]$. Chondrocytes have been proven to have the ability to create a chondrogenic niche for BMSCs to undergo chondrogenesis [12]. Besides that, chondrogenic differentiation of BMSCs could also be achieved using induction media containing growth factors and cytokines [7-9] or by using chondrocyte-conditioned media to supplement BMSCs cultured in a $3 \mathrm{D}$ scaffold $[13,14]$.

Transforming growth factor- $\beta$ (TGF- $\beta$ ) is a commonly used growth factor in induction medium for the chondrogenic induction of MSCs $[1,7,15-17]$. Although TGF- $\beta$ is a potent chondroinductive agent, it also directs cells to adopt a hypertrophic phenotype in vitro, in which cells will terminally differentiate, undergo apoptosis, and produce a matrix with inferior mechanical properties [18]. In terms of the regeneration of articular cartilage, it is a challenge to retain a stable chondrocyte phenotype when it becomes hypertrophic [19]. Chondrocyte hypertrophy is also considered a sign of OA where chondrocyte hypertrophy-like changes play a role in early and late stage OA [20].

Bearing all of that in mind, a successful tissue engineering approach could promote the robust chondrogenic differentiation of MSC and cartilage matrix synthesis without hypertrophy, particularly when challenged by the inflammatory and hypoxic microenvironment of the injured or osteoarthritic joint. Thus, the aim of this study was to analyse and compare the potential of chondrocyte condition medium and conventional induction medium to differentiate BMSCs into chondrocyte lineage.

\section{Material and methods}

This study was approved by the Universiti Kebangsaan Malaysia Research Ethics Committee (certificate of approval no. FF-2014-215).

\section{Isolation of chondrocytes and bone marrow stem cells (BMSCs)}

Human chondrocytes were isolated from redundant cartilage of 43-75 years old consented patients from Southeast Asia; undergoing total knee replacement surgery. Only the normal cartilage samples proximal to the affected area were processed within $24 \mathrm{~h}$ of surgery. The cartilage was finely minced and immediately digested with $0.6 \%$ Collagenase type II for 3 to $4 \mathrm{~h}$, at $37^{\circ} \mathrm{C}$ under continuous agitation. After that, the suspension of digested cartilage was centrifuged I and the pellet containing isolated cells were seeded with Chondrocyte Medium (PromoCell, USA). For BMSCs, aspirated bone marrow was diluted at a 1:1 ratio with F12:DMEM (FD) medium supplement with $10 \%$ foetal bovine serum (FBS). The diluted bone marrow was layered over $10 \mathrm{ml}$ of FicollPaque, and centrifuged at 2,500 rpm for $30 \mathrm{~min}$. Mononuclear cells were collected at the interface, and then suspended in PBS before being centrifuged at 5,000 rpm for $15 \mathrm{~min}$. The pellet was then resuspended in FD medium supplemented with $10 \%$ FBS and was seeded in a 6 -well plate. Once both cells were confluent, they were sub-cultured at 5,000 cells/ $\mathrm{cm}^{2}$ until passage 3 . All culture media were changed on alternate days.

\section{Collection of chondrocyte-conditioned medium (CCM)}

Confluent chondrocyte culture was used to prepare CCM. Cells were washed with Dulbecco Phosphate Buffer Saline (DPBS) twice and then supplemented with FD without FBS. The CCM was collected after 48 and $72 \mathrm{~h}$ of culture. This step was repeated for passage 1 to $3 . \mathrm{CCM}$ was stored at $-20^{\circ} \mathrm{C}$ until use. The ratio of conditioned medium that yielded the highest chondrogenic differentiation was examined by culturing the MSCs for 7, 14, and 21 days in FD medium containing 25\% and $50 \%(\mathrm{v} / \mathrm{v})$ conditioned medium.

\section{Bicinchoninic acid (BCA) assay}

Culture supernatants were collected from each sample and protein quantification was performed using the BCA assay kit 
(SIGMA). The absorbance was read at $562 \mathrm{~nm}$ using a spectrophotometer (BioTek PowerWave XS, Biotek, USA). The standard curve was plotted using BSA protein standards from 0 to $1000 \mathrm{mg} / \mathrm{ml}$ and the amount of protein in CCM was calculated.

\section{Chondrogenic induction of BMSCs}

BMSCs were induced using CCM and chondrogenic induction medium (CIM). Non-induced BMSCs were used as a control. For the induction, $25 \%$ and $50 \%(\mathrm{v} / \mathrm{v}) \mathrm{CCM}$ in FD medium were supplemented to BMSCs. Chondrogenic Induction Medium (CIM) was prepared as described previously [1]. Confluent BMSCs at passage 3 were induced with CIM or CCM for 21 days and the media were changed every 3 days. The induced BMSCs with CIM and CCM are hereafter referred to as iCIM and iCCM, respectively.

\section{Immunocytochemical staining}

Cells were washed with DPBS prior to fixing with $4 \%$ paraformaldehyde (Sigma-Aldrich) for $30 \mathrm{~min}$. Then, the cells were permeabilised for 20 min with $0.5 \%$ Triton X-100 solution (Sigma-Aldrich), and blocked with 10\% goat serum (Sigma-Aldrich) for $1 \mathrm{~h}$ at $37^{\circ} \mathrm{C}$. The cells were subsequently incubated with 1:200 rabbit anti-collagen 1 (TA309096; OriGene, USA) and 1:200 mouse anti-collagen II antibodies (ab185430; Abcam, USA) overnight at $4{ }^{\circ} \mathrm{C}$. On the following day, the cells were washed before being incubated with Alexa Fluor 594 goat anti-rabbit IgG (Invitrogen, USA) and Alexa Fluor 488 goat anti-mouse (Invitrogen, USA), respectively, for $1 \mathrm{~h}$ at $37^{\circ} \mathrm{C}$. Then, nuclei were counterstained with 1:20,000 4',6-diamidino-2-phenylindole (DAPI) before being observed using a Nikon Eclipse Ti fluorescence microscope (Nikon, Japan). Immunofluorescence staining was performed on 7, 14, and 21 days post-induction of BMSCs.

\section{Safranin-O staining}

Cells were washed with DPBS prior to fixation with $10 \%$ formalin. After $10 \mathrm{~min}$, the fixative was removed, and each well was washed twice with DPBS. Then, fast green solution (Dako, Denmark) was added to the well for $5 \mathrm{~min}$ before rinsing with $1 \%$ acetic acid for $15 \mathrm{sec}$. Then, $0.1 \%$ SafraninO (Sigma-Aldrich) solution was added to the well for $5 \mathrm{~min}$. Finally, the cells were washed twice with DPBS and observed under Olympus CK40 inverted microscope (Olympus, Germany).

\section{Toluidine blue staining}

Cells were washed with DPBS prior to fixation with $10 \%$ formalin. After $10 \mathrm{~min}$, the fixative was removed, and each well was washed twice with DPBS. Next, 0.04\% toluidine blue (Gainland UK) was added to the well for $1 \mathrm{~h}$ at room temperature. Lastly, the cells were washed twice with DPBS and observed under Olympus CK40 inverted microscope (Olympus, Germany).

\section{Quantitative real-time PCR (qRT-PCR)}

After 21 days of culture and induction, the expression of collagen type I (COL1), collagen type II (COL2), collagen type $\mathrm{X}$ (COLX), aggrecan core protein $(A C P)$, and sex-determining region $\mathrm{Y}$ box 9 (SOX9) genes in chondrocytes (control), BMSCs (control), iCCM and iCIM was evaluated using real-time RT-PCR. The cell was trypsinized and centrifuged at $5000 \mathrm{rpm}$ for 5 minutes at $37^{\circ} \mathrm{C}$. The cells pellet was suspended and total RNA was extracted using column method by Qiagen RNeasy Mini kit (Qiagen, Germany) and quantified with spectrophotometer (BioTek PowerWave XS, Biotek, USA). RNA integrity was ensure by performing agarose gel electrophoresis together with Olerup SSP GelRed (CareDx, USA). A 20- $\mu$ l aliquot of first-strand cDNA was synthesizes using the QuantiNova ${ }^{\mathrm{TM}}$ Reverse Transcription kit (Qiagen, Germany) according to the manufacturer's protocol. QuantiNova ${ }^{\circledR}$ SYBR ${ }^{\circledR}$ Green PCR (Qiagen, Germany) was used for quantitative polymerase chain reaction (qPCR) analysis using $2 \mu \mathrm{l}$ of each reverse-transcribed sample. The assay was run in a StepOne Plus Real-Time thermal cycler (Applied Biosystems, USA) with the following PCR cycling conditions: initial denaturation for $2 \mathrm{~min}$ at $95^{\circ} \mathrm{C}$, followed by 40 cycles of denaturation for $5 \mathrm{sec}$ at $95^{\circ} \mathrm{C}$, then annealing/extension for $10 \mathrm{~min}$ at $60^{\circ} \mathrm{C}$ for all the primer pairs. The assay was followed by a melt curve analysis to ensure PCR product specificity. Table 1 shows the primer sequence used for RT-PCR following previous studies [42-44]. Glyceraldehyde-3-phosphate dehydrogenase (GAPDH) was used as the reference gene. The data were analyzed using The StepOne ${ }^{\mathrm{TM}}$ Software. For gene expression quantitation, the comparative $\mathrm{Ct}$ method was used. The $\mathrm{Ct}$ values of the gene of interest were normalized to GAPDH. The $\mathrm{Ct}$ value was calculated when the fluorescence of the sample exceeded threshold level. 
Table 1. Description of the primers used in the quantitative RT-PCR analysis

\begin{tabular}{|c|c|c|}
\hline Gene & Forward primer $\left(5^{\prime}\right.$ to $\left.3^{\prime}\right)$ & Reverse primer ( $5^{\prime}$ to $\left.3^{\prime}\right)$ \\
\hline COL 1 & AAGGCTTCAAGGTCCCCCTGGTG & CAGCACCAGTAGCACCATCATTTC \\
\hline $\operatorname{COL} 2$ & GGCAATAGCAGGTTCACGTACA & CGATAACAGTCTTGCCCCACTT \\
\hline $\operatorname{coL} x$ & CGTCTTCAGCGCCAAGC & CGCCATTCTTCACCAGATCAAA \\
\hline $\mathrm{ACP}$ & CATTCGGCGGACAAATTAGATG & CCTACAAACGCAGACTACAGAA \\
\hline SOX9 & CTGAGTCATTTGCAGTGTTTTCT & CATGCTTGCATTGTTTTTTGTGT \\
\hline GAPDH & GGCGATGCTGGCGCTGAGTAC & TGGTTCACACCCATGACGA \\
\hline
\end{tabular}

$\mathrm{F}$, forward (sense) primer; $\mathrm{R}$, reverse (anti-sense) primer

\section{Statistical analysis}

Data are shown as mean of 3 biological samples \pm SEM $(\mathrm{n}=3)$. The comparison of the mean between groups was assessed with one-way ANOVA test of variance. $P \leq 0.05$ was considered significantly different.

\section{Results}

\section{Protein concentration in CCM}

Protein concentration was found to be significantly higher in passage $3 \mathrm{CCM}(1023.22 \pm 55.85 \mu \mathrm{g} / \mathrm{ml}$ and $1108.64 \pm 7.82 \mu \mathrm{g} /$ $\mathrm{ml}$ for $48 \mathrm{~h}$ and $72 \mathrm{~h}$ incubation, respectively) compared to the CCM from other passages (Figure 1). There is no significant difference between the amounts of protein for CCM collected $48 \mathrm{~h}$ and $72 \mathrm{~h}$ post-culture in all passages. Therefore, medium that has been conditioned for $72 \mathrm{~h}$ using passage 3 chondrocytes was chosen for further evaluation.

\section{Cellular morphology during chondrogenic induction}

As shown in Figure 2, BMSCs exhibited fibroblastic shape, while chondrocytes have more rounded and polygonal shapes with small extensions. Both type of cells grew in monolayers when cultured in their respective culture medium (FD $+10 \%$ FBS and Chondrocyte medium). The iCIM started to form aggregates or clumps as early as day 7. Meanwhile, iCCM started to grow compactly in several spots; however, there was no prominent clump formation observed.

\section{Immunocytochemistry analysis}

Type 1 collagen was expressed prominently in every time point for all groups. Meanwhile, Type 2 collagen was not expressed in the BMSC group throughout the culture period (Figure 3). Chondrocytes expressed collagen type 2; however, the expression became weaker with increasing culture time. For the ICIM and ICCM group, the treatment caused the induced BMSCs to express collagen type 2 throughout the culture period.

\section{Safranin-O staining}

iCCM was stained with Safranin-O to test for their GAG synthesis ability. Non-induced BMSCs showed negative staining for Safranin-O while BMSCs induced with CCM and CIM showed the positive staining of Safranin-O as early as day 7 (Figure 4).

\section{Toluidine blue staining}

BMSCs showed no positive staining for toluidine blue. The iCIM and iCCM 50\% on day 21 showed the prominent staining of toluidine blue similar to chondrocytes which showed the formation of proteoglycans and glycosaminoglycans by the induced cell (Figure 5).

\section{Quantitative RT-PCR}

The gene expression for type I, II, and X collagens, Sox9, and aggrecan core protein (a proteoglycan composed of GAGs) on day 21 was evaluated. As shown in Table 2, chondrocytes had the higher expression of collagen type 2 compared to BMSCs, $\mathrm{iCIM}$ and $\mathrm{iCCM}$. There was no significant difference between the iCIM and ICCM group with regard to collagen type 2 expression; however, both groups expressed higher levels of collagen type 2 when compared to BMSCs. ACP expression was significantly higher in chondrocytes compared to BMSCs, but there were no significant differences in ACP expression 


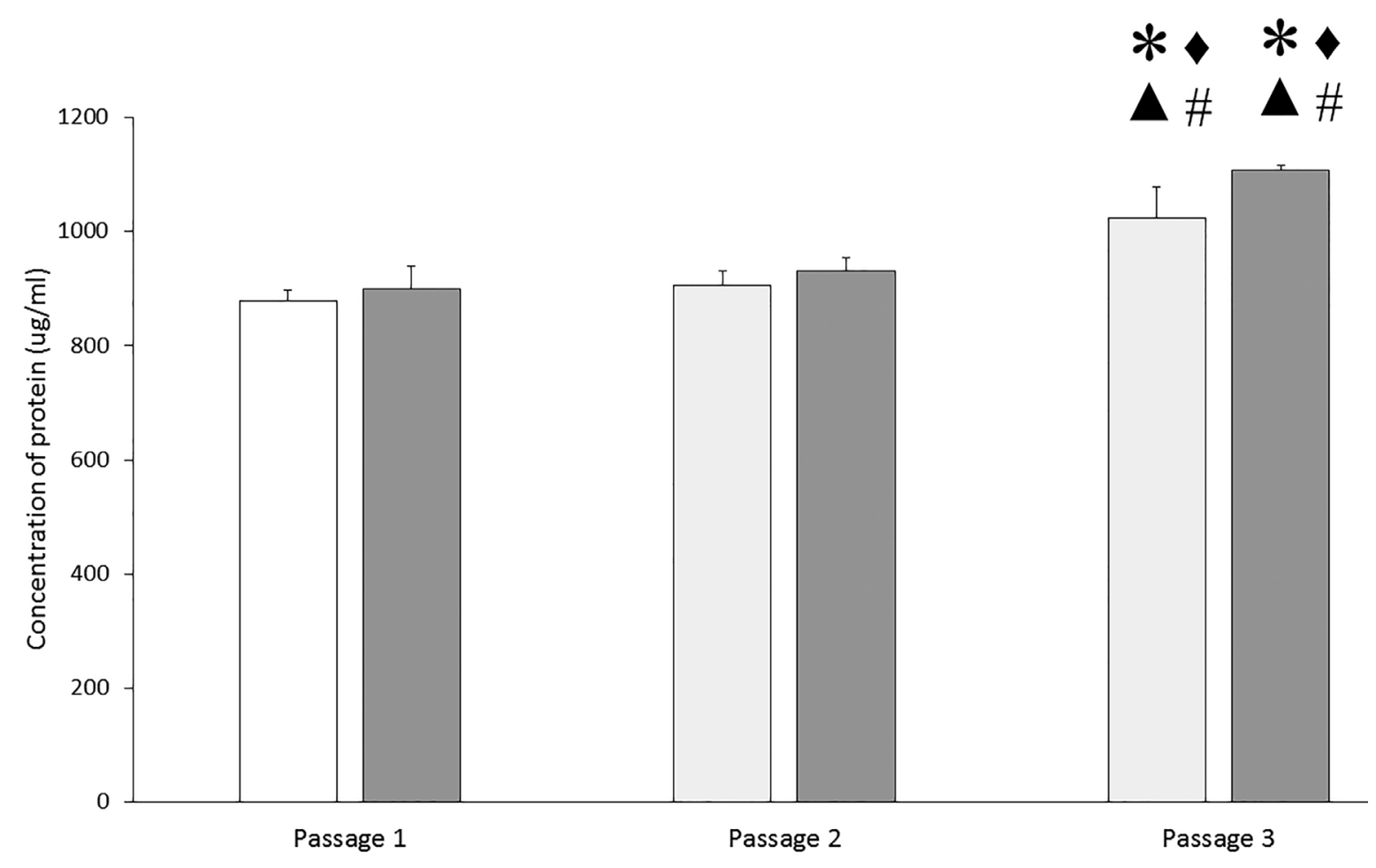

Figure 1. Protein concentrations for iCCM for every passage at different collection time points. There are no significant differences in the passage 3 protein concentration between $48 \mathrm{~h}$ (unfilled bars) $(1023.22 \pm 55.85 \mu \mathrm{g} / \mathrm{ml})$ and $72 \mathrm{~h}$ (gray bars) $(1108.64 \pm 7.82 \mu \mathrm{g} / \mathrm{ml})$. *significant differences with P1 (48 h), $\Delta$ significant differences with P1 (72 h), significant differences with P2 (48 h), and \#significant differences with P2 (72 h).
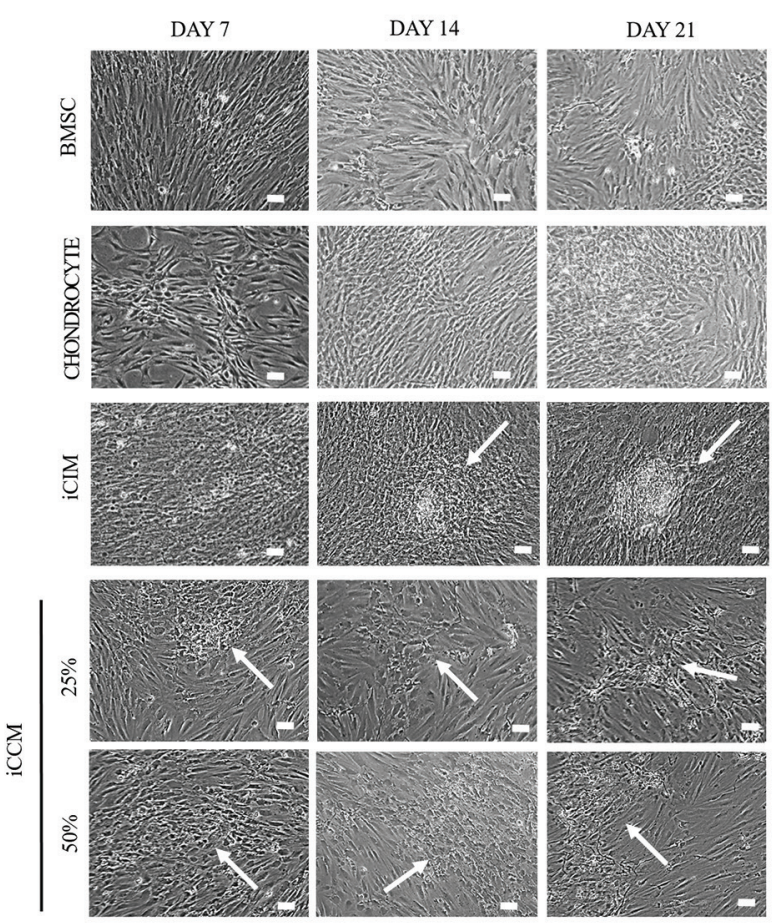

Figure 2. Morphology of BMSCs, chondrocyte and ICIM and iCCM. Arrows show the formation of aggregates. Bars represent $100 \mu \mathrm{m}$. between chondrocytes, iCIM and iCCM. Expression of the SOX9 gene in chondrocytes, ICIM and ICCM was higher compared to BMSCs. Besides that, iCIM and iCCM have significantly higher SOX9 expression than chondrocytes. The relative expression of collagen type 1 was the highest in BMSCs. CCM caused a reduction of collagen type 1 relative expression compared to non-induced BMSCs. For collagen type 10, BMSCs, iCIM and iCCM had lower expression levels compared to chondrocytes. The expression of collagen type 10 in iCCM was significantly higher compared to in iCIM and BMSCs.

\section{Discussion}

Cultured cells are known to release various growth factors into the culture medium. Several studies have shown the use of chondrocyte-conditioned medium for chondrogenesis purposes, with different methods of cultured medium conditioning using scaffolds or with different chondrocyte sources i.e. bovine and sheep [14, 21, 22]. However, none used conditioned medium from monolayer human primary chondrocytes for articular cartilage regeneration. In this 


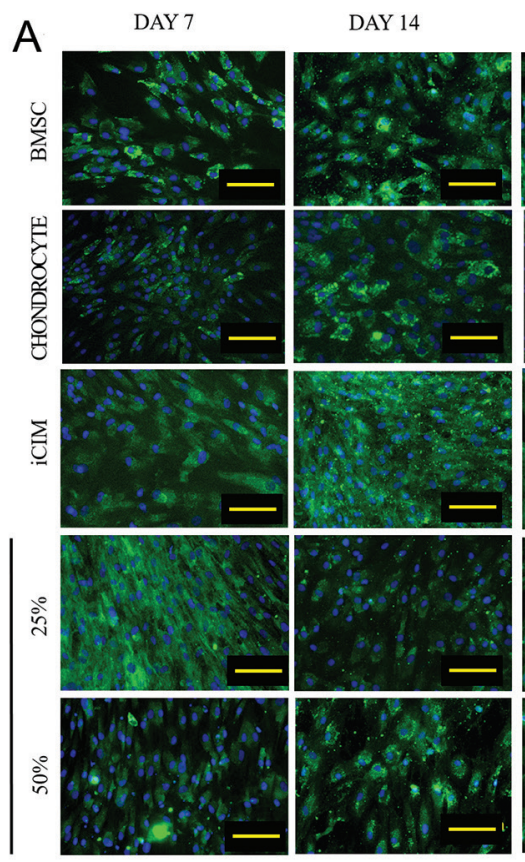

DAY 21
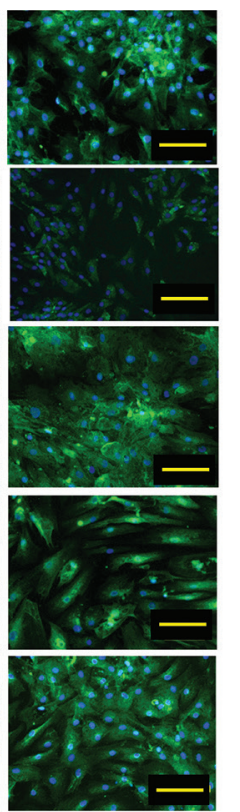

B

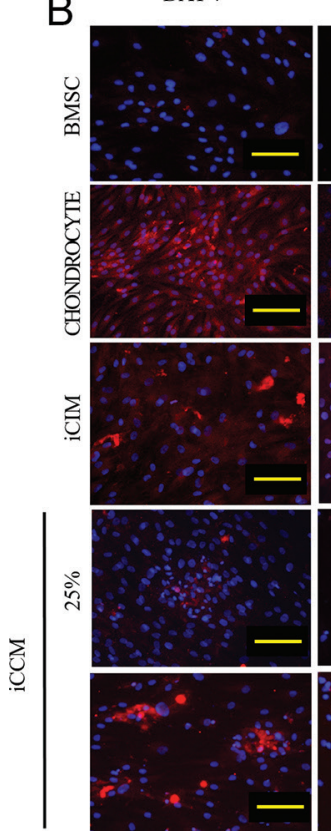

DAY 14

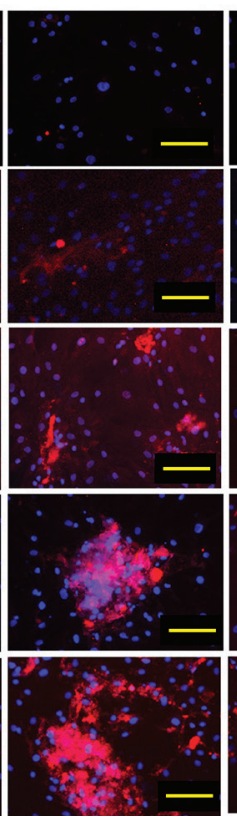

DAY 21

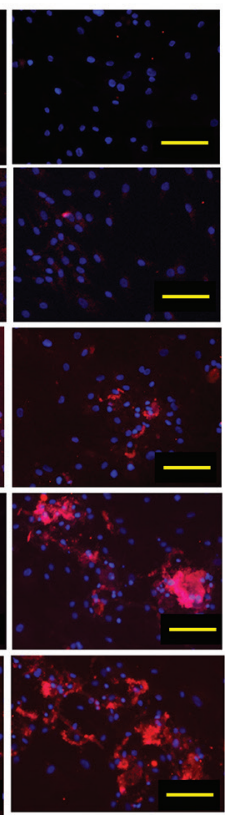

Figure 3. Immunofluorescence images of BMSCs, Chondrocytes, and ICIM and iCCM on days 7, 14, and 21. Cells were stained for (A) collagen 1 (green) and (B) collagen 2 (red). Nuclei were stained with DAPI (blue). Yellow bar represents $100 \mu \mathrm{m}$.
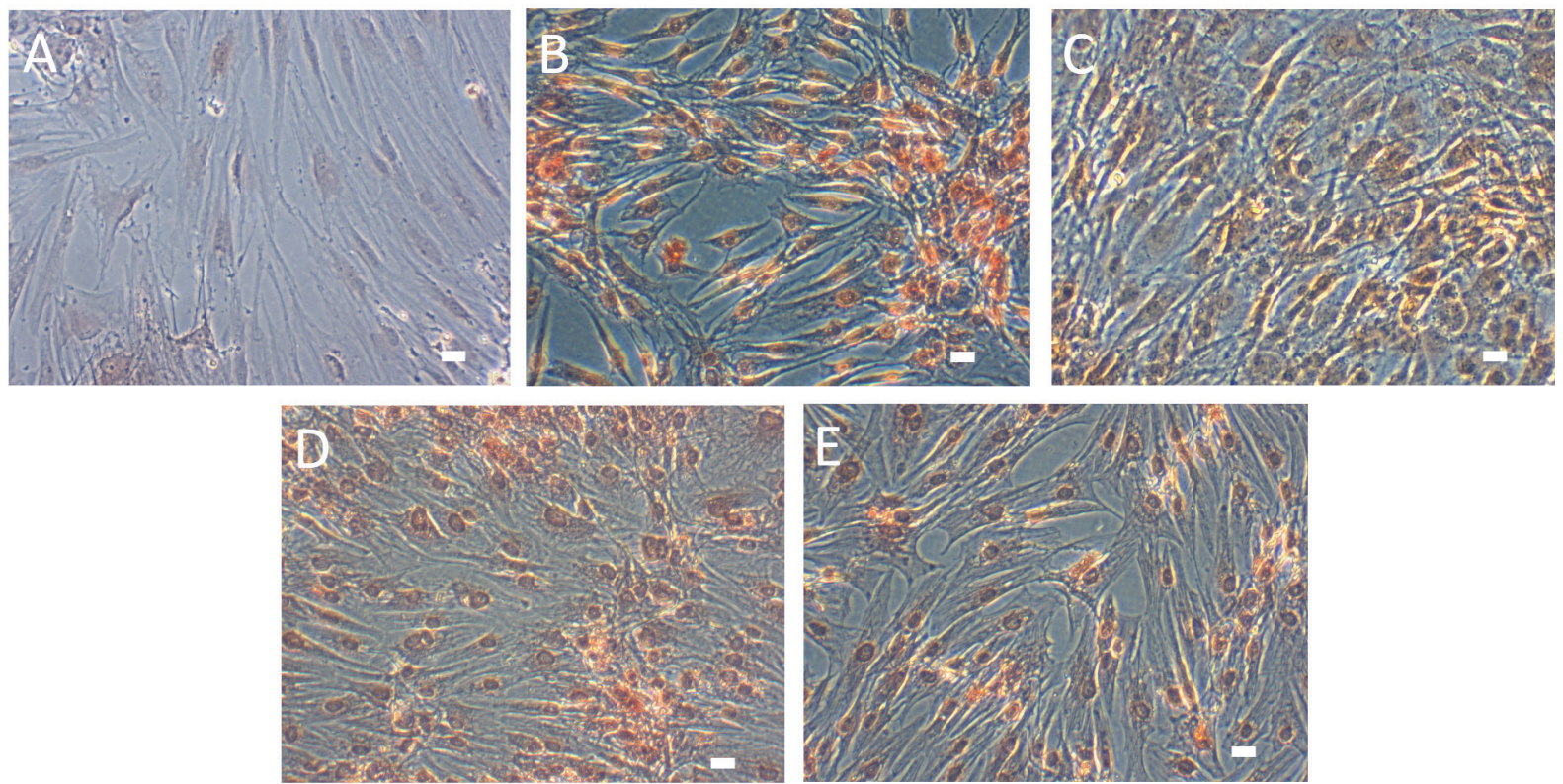

Figure 4. iCCM and chondrocytes showed positive staining for Safranin-O, indicating that the synthesis of the extracellular matrix (GAG) happened as early as day 7. (A) BMSCs, (B) Chondrocytes, (C) iCIM, (D) iCCM 25\%, and (E) iCCM 50\%. Bars represent $100 \mu \mathrm{m}$.

study, the optimisation of several experimental parameters for chondrocyte-conditioned medium (CCM) such as chondrocyte passage, conditioned medium collection time and CCM:fresh media ratio for BMSC chondrogenic induction was performed.
BMSCs were the negative control, whereas chondrocytes and iCIM were the positive control for induction.

In order to further select the optimum conditioned medium, protein quantification was performed to quantitate 


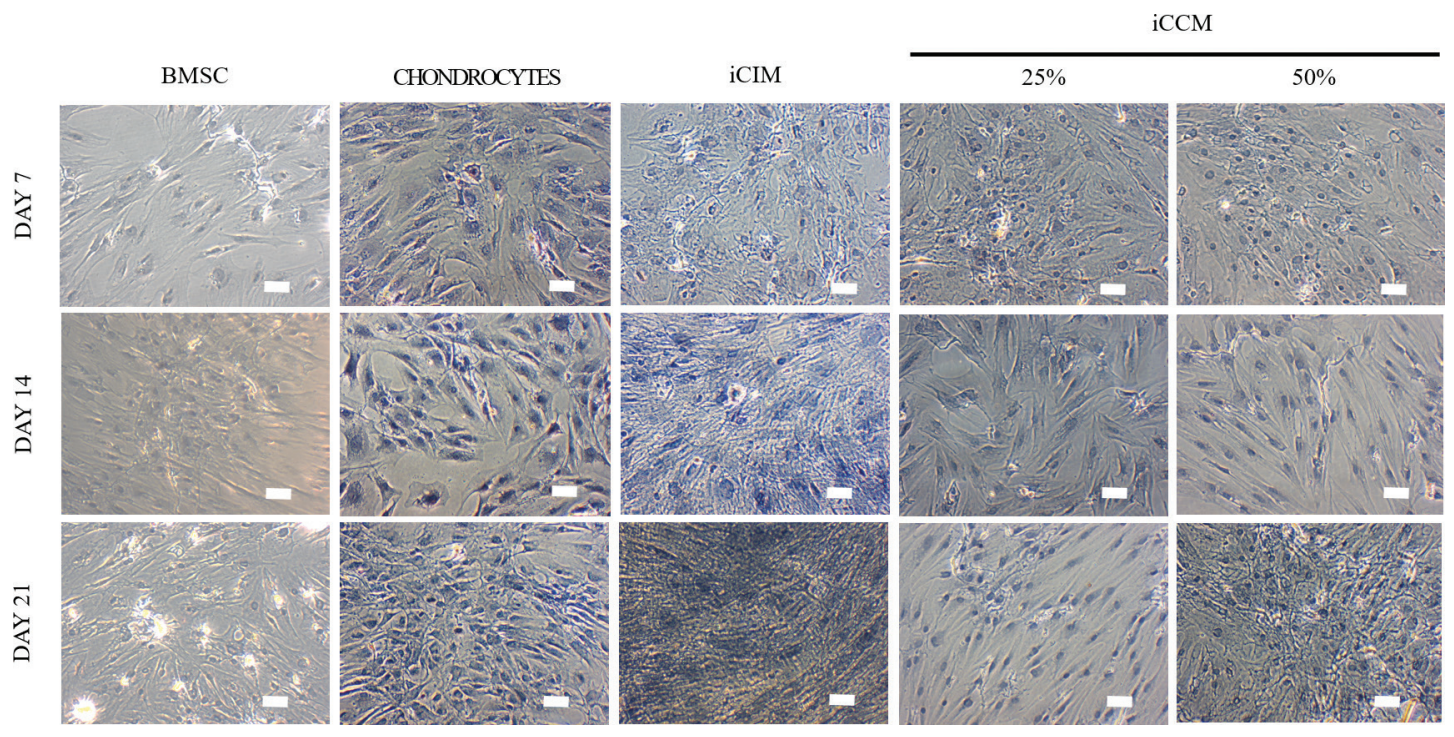

Figure 5. Toluidine blue staining of BMSCs, Chondrocytes, iCIM and tested groups of iCCM at days 7, 14 and 21. Bars represent $100 \mu \mathrm{m}$.

Table 2. COL 2, ACP, SOX9, COL 1 and $C O L 10$ gene expression of BMSCS, iCIM, iCCM, and chondrocytes

\begin{tabular}{lccccc}
\hline & COL 2 & ACP & SOX9 & COL 1 & COL 10 \\
\hline BMSCs & $0.000110 \pm 0.000067^{*}$ & $0.0279 \pm 0.0377^{*}$ & $0.000127 \pm 0.000159^{*}$ & $0.584 \pm 0.181^{*}$ & $0.000118 \pm 0.000102^{*}$ \\
iCIM & $0.001068 \pm 0.000882^{\dagger}$ & $0.0636 \pm 0.0711$ & $0.024188 \pm 0.041229^{\dagger}$ & $0.417 \pm 0.404$ & $0.000268 \pm 0.000212^{+, \#}$ \\
iCCM & $0.001047 \pm 0.000929^{\ddagger}$ & $0.0467 \pm 0.0408$ & $0.003450 \pm 0.005637^{\ddagger}$ & $0.173 \pm 0.283$ & $0.000736 \pm 0.000183^{\ddagger, 5}$ \\
Chondrocytes & $0.154506 \pm 0.006891$ & $0.0952 \pm 0.0038$ & $0.544180 \pm 0.098256$ & $0.160 \pm 0.028$ & $0.060961 \pm 0.049591$ \\
\hline
\end{tabular}

*a significant difference in gene expression between BMSCs vs chondrocytes. ${ }^{\dagger}$ a significant difference in gene expression between iCIM vs chondrocytes. ${ }^{\ddagger}$ a significant difference in gene expression between iCCM vs chondrocytes. " a significant difference in gene expression between iCCM vs iCIM. sa significant difference in gene expression between iCCM vs BMSCs.

the amount of protein released into the medium. Based on these results, CCM that had been conditioned for $72 \mathrm{~h}$ at passage 3 was chosen for further analysis as it secreted more protein compared to the other time points and passages. Late passage cells have been observed to have increased amounts of protein compared to earlier passages [23]. However, the compositions of the proteins are also reported to be different according to passage, which also need to be taken into consideration $[23,24]$.

BMSCs that have been induced with CIM formed clumps, which is one of the early signs of chondrogenesis for induced BMSCs [1]. However, for iCCM, the cells only started to form compact layer of cells and no clump or aggregate was observed in the culture. These differences might be due to the higher number of cells that were present in the iCIM group compared to iCCM due to the presence of growth factors, especially TGF 33 in CIM, which helps with cell proliferation [25].

Immunocytochemistry (ICC) analysis showed that the expression of collagen type 2 is lower throughout time from day 7 to day 21 for all of the tested groups. This result is similar to those reported by Mardani et al., where the expression of collagen type 2 reduced over time [26]. It has been well-documented that prolonged culture will cause the cells to express type 1 collagen. Other than that, $50 \%$ conditioned medium for both iCCM groups showed higher expression compared to the $25 \%$ conditioned medium. Therefore, $50 \%$ conditioned medium is presumed to be more effective in inducing BMSCs into chondrocytes. This finding is also supported by several previous studies, which showed that a 50:50 ratio of conditioned medium and fresh medium promoted the differentiation of BMSCs into chondrocytes [14, 27]. However, ICC alone could not be used to conclude a successful chondrogenesis attempt [28]; therefore, gene expression analysis using toluidine blue and safranin-O staining was performed.

Safranin-O and toluidine blue staining are well-known stains used to characterise cartilage tissue. They are cationic dyes that stain proteoglycans and glycosaminoglycans, which are abundant proteins in the ECM of chondrocytes. All of the tested groups were positive for Safranin-O staining and toluidine blue staining as early as day 7 , which suggests that 
induced bone marrow has started to produce proteoglycans and glycosaminoglycans. Naturally, proteoglycans and glycosaminoglycans are produced by chondrocytes and secreted into the ECM or plasma membrane, or stored in secretory granules in the cells [29]. This characteristic explains the positive staining all around the nucleus inside cells. However, the staining intensity is not high compared to the positively stained cartilage tissue. Safranin-O staining is normally used to stain cartilage tissue $[1,15,30]$ and seldom on monolayer cells on tissue culture plates [31]. The abundant amount of proteoglycans and glycosaminoglycans in cartilage tissue contributes to the bright red staining observed in the tissue.

Based on cell morphology, protein quantification, ICC and Safranin-O staining, the best conditioned medium that induced bone marrow differentiation into chondrocytes was $50 \%$ of the $72 \mathrm{hr}$ CCM at passage 3 . The chosen conditioned medium was used to evaluate its chondrogenesis ability through gene expression analysis.

Endochondral ossification is a process of bone formation which starts with the condensation of MSCs that differentiate into chondrocytes, which later form a cartilage template that is replaced by bone minerals. Chondrogenesis is part of the process of endochondral ossification. MSCs during condensation will express SOX9, which is an important regulatory factor in chondrogenesis [32]. After that, these immature chondrocytes will express the transcription factor SOX9, collagen type 2, and aggrecan. All of these markers are chondrocyte differentiation markers. In this study, iCCM expressed all of the chondrocyte differentiation markers and it was observed that there were no significant differences between ICIM and ICCM in terms of the expression of chondrogenic marker genes: collagen type II and the aggrecan core protein. Both groups showed the higher expression of those chondrogenic markers compared to BMSCs. This shows that CCM has the same ability as iCIM to induce bone marrow into chondrocytes.

Subsequently, during the endochondral ossification process, chondrocyte pre-hypertrophy starts and cells eventually enter the early hypertrophic chondrocyte stage where collagen type 10 is expressed. Afterwards, the expression of SOX 9 and collagen type 2 decreases. Finally, chondrocytes proceed into late hypertrophic status [33]. It was observed that CCM caused the significant down-regulation of collagen type 1 and collagen type $\mathrm{X}$ genes in bone marrow. Collagen type $\mathrm{X}$ is expressed by hypertrophic chondrocytes during endochondral ossification, which eventually changes chondrocytes into osteoblasts and osteocytes [19]. Chondrocytes in the human body exist in the extracellular matrix (ECM) where they live in the chondron [34]. Chondrocyte metabolic activities and phenotypes are regulated by the ECM, which also provides mechanical protection [35]. However, under in vitro conditions, chondrocytes or induced BMSCs lose their interaction with the 3-dimensional surrounding, thereby changing their phenotype and gene expression patterns [36-38].

In this study, we investigated the potential of CCM to induce chondrogenesis in BMSCs. Induction protocols should be improved to produce a stable and non-hypertrophic cartilage tissue. It was reported that the culture of chondrocytes produces soluble factors that suppress the terminal differentiation of chondrocytes in vitro [39]. Therefore, in the future, combinations of these two types of induction medium might help to produce better chondrogenic-induced BMSCs.

Besides that, culturing chondrocytes in a $3 \mathrm{D}$ environment mimicking the native conditions, e.g. hypoxic conditions, pellet culture or in a scaffold, might help to further induce BMSCs towards chondrocytes, as the cells were cultured under similar conditions as native cells in the human body [1, 13, 35, 40, 41]. In addition, secretome profiling of the conditioned medium should be performed as it is important to identify the secreted factors and understand the underlying mechanism that contributes to the phenotypic changes that the cells go thorough in in vitro culture. Identifying the chondrogenesis mediators could help to improve the existing approach, thereby helping to develop new techniques for acellular cartilage repair.

\section{Conclusion}

In this study, the conditions for the collection of CCM have been optimised; it was shown that the best conditioned medium that supports the induction of bone marrow into chondrocytes was $72 \mathrm{hr}$ medium with a $50 \%$ ratio of CCM to fresh medium at passage 3. CCM was comparable with the known induction medium CIM, in terms of inducing bone marrow into chondrocytes. Thus, CCM has potential for the in vitro and in vivo induction of BMSCs into chondrocytes for future therapeutic applications.

Author contributions. RAR, YL, SRC, NHMY, ABS, and RBHI contributed substantially to the conception and design of the study. RAR acquired the data. RAR, YL, SRC, and RBHI analysed and interpret the data. RAR drafted the manuscript. YL, SRC, NHMY, ABS, and RBHI critically revised the manuscript. All the authors approved the final version submitted for publication and take responsibility for the statements made in the published article.

Acknowledgments. This study was supported by the Ministry of Higher Education Malaysia Fundamental Research Grant Scheme [FRGS/1/2013/SG05/UKM/01/3]; and the National 
University of Malaysia (UKM) Arus Perdana grant [AP 2013015].

Conflict of interest statement. The authors have each completed and submitted an International Committee of Medical Journal Editors form for disclosure of Potential Conflicts of Interest. None of the authors has any conflict of interest to disclose.

\section{References}

[1] Ude CC, Sulaiman SB, Min-Hwei N, Hui-Cheng C, Ahmad J, Yahaya NM, et al. Cartilage regeneration by chondrogenic induced adult stem cells in osteoarthritic sheep model. PLoS One. 2014; 9:e98770.

[2] Ganasegeran K, Menke JM, Challakere Ramaswamy VM, Abdul Manaf R, Alabsi AM, Al-Dubai SAR. Level and determinants of knowledge of symptomatic knee osteoarthritis among railway workers in Malaysia. Biomed Res Int. 2014; 2014. doi: https://doi. org/10.1155/2014/370273.

[3] Zhang Y, Jordan JM. Epidemiology of osteoarthritis. Clin Geriatr Med. 2010; 26:355-69.

[4] Fox AJS, Bedi A, Rodeo SA. The basic science of articular cartilage: structure, composition, and function. Sport Heal A Multidiscip Approach. 2009; 1:461-8.

[5] Kolettas E, Muir HI, Barrett JC, Hardingham TE. Chondrocyte phenotype and cell survival are regulated by culture conditions and by specific cytokines through the expression of Sox- 9 transcription factor. Rheumatology. 2001; 40:1146-56.

[6] Indrawattana N, Chen G, Tadokoro M, Shann LH, Ohgushi H, Tateishi T, et al. Growth factor combination for chondrogenic induction from human mesenchymal stem cell. Biochem Biophys Res Commun. 2004; 320:914-9.

[7] Alfaqeh HH, Hui CK, Saim AB, Idrus RB. Growth medium with low serum and transforming growth factor beta 3 promotes better chondrogenesis of bone marrow-derived stem cells in vitro and in vivo. Saudi Med J. 2011; 32:640-1.

[8] Al Faqeh H, Hamdan BMYN, Chen HC, Aminuddin BS, Ruszymah $\mathrm{BHI}$. The potential of intra-articular injection of chondrogenicinduced bone marrow stem cells to retard the progression of osteoarthritis in a sheep model. Exp Gerontol. 2012; 47:458-64.

[9] Hamid AA, Idrus RBH, Saim A Bin, Sathappan S, Chua K-H. Characterization of human adipose-derived stem cells and expression of chondrogenic genes during induction of cartilage differentiation. Clinics. 2012; 67:99-106.

[10] Zhang L, Li Q, Zhou G, Liu Y, Liu W, Zhang W, et al. Co-culture of microtic chondrocyte with BMSC to generate tissue engineered cartilage. Tissue Eng Part A. 2011. doi: 10.1089/ten.TEA.2011.0443

[11] Meretoja V V, Dahlin RL, Kasper FK, Mikos AG. Enhanced chondrogenesis in co-cultures with articular chondrocytes and mesenchymal stem cells. Biomaterials. 2012; 33:6362-9.

[12] Liu X, Sun H, Yan D, Zhang L, Lv X, Liu T, et al. In vivo ectopic chondrogenesis of BMSCs directed by mature chondrocytes. Biomaterials. 2010; 31:9406-14.

[13] Liu J, Liu X, Zhou G, Xiao R, Cao Y. Conditioned medium from chondrocyte/scaffold constructs induced chondrogenic differen- tiation of bone marrow stromal cells. Anat Rec Adv Integr Anat Evol Biol. 2012; 295:1109-16.

[14] Alves da Silva ML, Costa-Pinto AR, Martins A, Correlo VM, Sol P, Bhattacharya $\mathrm{M}$, et al. Conditioned medium as a strategy for human stem cells chondrogenic differentiation. J Tissue Eng Regen Med. 2015; 9:714-23.

[15] Ude CC, Ng MH, Chen CH, Htwe O, Amaramalar NS, Hassan S, et al. Improved functional assessment of osteoarthritic knee joint after chondrogenically induced cell treatment. Osteoarthr Cartil. 2016; 23:1294-306.

[16] Tuli R, Tuli S, Nandi S, Huang X, Manner PA, Hozack WJ, et al. Transforming growth factor-beta-mediated chondrogenesis of human mesenchymal progenitor cells involves $\mathrm{N}$-cadherin and mitogen-activated protein kinase and Wnt signaling cross-talk. J Biol Chem. 2003; 278:41227-36.

[17] Joyce ME, Roberts AB, Sporn MB, Bolander ME. Transforming growth factor-beta and the initiation of chondrogenesis and osteogenesis in the rat femur. J Cell Biol. 1990; 110:2195-207.

[18] Cooke ME, Allon AA, Cheng T, Kuo AC, Kim HT, Vail TP, et al. Structured three-dimensional co-culture of mesenchymal stem cells with chondrocytes promotes chondrogenic differentiation without hypertrophy. Osteoarthr Cartil. 2011; 19:1210-8.

[19] Shen G. The role of type X collagen in facilitating and regulating endochondral ossification of articular cartilage. Orthod Craniofac Res. 2005; 8:11-7.

[20] Van der Kraan PM, Van den Berg WB. Chondrocyte hypertrophy and osteoarthritis: role in initiation and progression of cartilage degeneration? Osteoarthr Cartil. 2012; 20:223-32.

[21] Hwang NS, Varghese S, Puleo C, Zhang Z, Elisseeff J. Morphogenetic signals from chondrocytes promote chondrogenic and osteogenic differentiation of mesenchymal stem cells. J Cell Physiol. 2007; 212:281-4.

[22] Zhao X, Hwang NS, Bichara DA, Saris DB, Malda J, Vacanti JP, et al. Chondrogenesis by bone marrow-derived mesenchymal stem cells grown in chondrocyte-conditioned medium for auricular reconstruction. J Tissue Eng Regen Med. 2017; 11:2763-73.

[23] Johnson JE. Aging and cell Structure. Springer US; [online] 2013. Available from: https://books.google.com.my/ books?id=6HrdBwAAQBAJ

[24] Petropoulos I, Conconi M, Wang X, Hoenel B, Brégégère F, Milner $\mathrm{Y}$, et al. Increase of oxidatively modified protein is associated with a decrease of proteasome activity and content in aging epidermal cells. J Gerontol A Biol Sci Med Sci. 2000; 55:B220-7.

[25] Lafontaine L, Chaudhry P, Lafleur M-J, Van Themsche C, Soares MJ, Asselin E. Transforming growth factor Beta regulates proliferation and invasion of rat placental cell lines. Biol Reprod. 2011; 84:553-9.

[26] Mardani M, Hashemibeni B, Ansar MM, Zarkesh Esfahani SH, Kazemi M, Goharian V, et al. Comparison between chondrogenic markers of differentiated chondrocytes from adipose derived stem cells and articular chondrocytes In vitro. Iran J Basic Med Sci. 2013; 16:763-73.

[27] Liu J, Liu X, Zhou G, Xiao R, Cao Y. Conditioned medium from chondrocyte/scaffold constructs induced chondrogenic differentiation of bone marrow stromal cells. Anat Rec Adv Integr Anat Evol Biol. 2012; 295:1109-16.

[28] Benoy IH, Elst H, Van der Auwera I, Laere S Van, Dam P van, Marck E Van, et al. Real-time RT-PCR correlates with immunocytochemistry for the detection of disseminated epithelial cells in bone 
marrow aspirates of patients with breast cancer. Br J Cancer. 2004; 91:1813-20.

[29] Esko JD, Kimata K, Lindahl U. Proteoglycans and sulfated glycosaminoglycans. In: Varki A, Cummings RD, Esko JD, et al., editors. Essentials of glycobiology. 2nd edition. Cold Spring Harbor (NY): Cold Spring Harbor Laboratory Press; [online] 2009. [cited 2019 Mar 10]. Available from: https://www.ncbi.nlm.nih.gov/books/ NBK1900/

[30] Schmitz N, Laverty S, Kraus VB, Aigner T. Basic methods in histopathology of joint tissues. Osteoarthr Cartil. 2010; 18:S113-6.

[31] Xu GJ, Lu ZH, Lin X, Lin CW, Zheng L, Zhao JM. Effect of JJYMD-C, a novel synthetic derivative of gallic acid, on proliferation and phenotype maintenance in rabbit articular chondrocytes in vitro. Brazilian J Med Biol Res. 2014; 47:637-45.

[32] Kozhemyakina E, Lassar AB, Zelzer E. A pathway to bone: signaling molecules and transcription factors involved in chondrocyte development and maturation. Development. 2015; 142:817-31.

[33] Li J, Dong S. The signaling pathways involved in chondrocyte differentiation and hypertrophic differentiation. Stem Cells Int. 2016; 2016.

[34] Zhang Z, Fan J, Becker KG, Graff RD, Lee GM, Francomano CA. Comparison of gene expression profile between human chondrons and chondrocytes: a cDNA microarray study. Osteoarthr Cartil. 2006; 14:449-59.

[35] Behonick DJ, Werb Z. A bit of give and take: the relationship between the extracellular matrix and the developing chondrocyte. Mech Dev. 2003; 120:1327-36.

[36] Urban JPG, Hall AC, Gehl KA. Regulation of matrix synthesis rates by the ionic and osmotic environment of articular chondrocytes. J Cell Physiol. 1993; 154:262-70.
[37] Malda J, Martens DE, Tramper J, van Blitterswijk CA, Riesle J. Cartilage tissue engineering: controversy in the effect of oxygen. Crit Rev Biotechnol. 2003; 23:175-94.

[38] Benz K, Breit S, Lukoschek M, Mau H, Richter W. Molecular analysis of expansion, differentiation, and growth factor treatment of human chondrocytes identifies differentiation markers and growth-related genes. Biochem Biophys Res Commun. 2002; 293:284-92.

[39] Jikko A, Kato Y, Hiranuma H, Fuchihata H. Inhibition of chondrocyte terminal differentiation and matrix calcification by soluble factors released by articular chondrocytes. Calcif Tissue Int. 1999; 65:276-9.

[40] Glennon-Alty L, Williams R, Dixon S, Murray P. Induction of mesenchymal stem cell chondrogenesis by polyacrylate substrates. Acta Biomater. 2013; 9:6041-51.

[41] Lee H-H, Chang C-C, Shieh M-J, Wang J-P, Chen Y-T, Young T-H, et al. Hypoxia enhances chondrogenesis and prevents terminal differentiation through PI3K/Akt/FoxO dependent anti-apoptotic effect. Sci Rep. 2013; 3:2683.

[42] Otto IA, Levato R, Webb WR, Khan IM, Breugem CC, Malda J. Progenitor cells in auricular cartilage demonstrate cartilage-forming capacity in 3D hydrogel culture. Eur Cell Mater. 2018; 35:132-50.

[43] Munirah S, Ruszymah BHI, Samsudin OC, Badrul A, Azmi B, Aminuddin BS. Autologous versus pooled human serum for articular chondrocyte growth. J Orthop Surg. 2008; 16:220-9.

[44] Dehne T, Karlsson C, Ringe J, Sittinger M, Lindahl A. Chondrogenic differentiation potential of osteoarthritic chondrocytes and their possible use in matrix-associated autologous chondrocyte transplantation. Arthritis Res Ther. 2009; 11:R133 